\title{
Multivariate Analyses and Characterization of Volatile Components in Citrus
}

\section{Species}

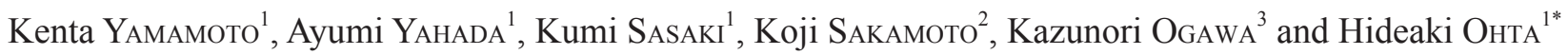 \\ ${ }^{1}$ Graduate School of Health and Nutrition Sciences, Nakamura Gakuen University, 5-7-1 Befu, Johnan-ku, Fukuoka 814-0198, Japan \\ ${ }^{2}$ Hirohsima Prefectural Food Technology Research Center, 12-70 Hijiyamahon-machi, Minami-ku, Hiroshima 732-0816, Japan \\ ${ }^{3}$ Department of Citriculture, National Research Institute of Fruit Tree Science, 485-6 Shimizuokitsu-cho, Shizuoka 424-0292, Japan
}

Received July 27, 2012; Accepted September 21, 2012

The composition of volatile components in 18 Citrus Archicitrus species, 17 Citrus Metacitrus species and two Fortunella species, according to the classification system established by Tanaka, was investigated using headspace gas chromatography-mass spectrometry. The composition of 48 volatile compounds in the juice of these species was determined, and principal component analysis was performed. Scores for the 1st and 2nd principal components are plotted as a scatter diagram. In Citrus Archicitrus, samples of Citrophorum, Cephacitrus and Aurantium formed individual groups, but others did not. In Citrus Metacitrus, samples of Acrumen were divided into two different groups. The composition of volatile components in citrus juice was consistent with each grouping of Tanaka's system, in particular, in the case of dividing the Citrus genus into the two subgenera Archicitrus and Metacitrus.

Keywords: Citrus, volatile component, headspace gas, SPME, characterization, classification

\section{Introduction}

Citrus fruits are cultivated in many countries the world over, and are widely consumed because of their preferable flavors. The majority of citrus fruits are used for the production of citrus juice. The aroma of citrus juice typically consists of several odor components, including esters, aldehydes, alcohols, ketones and hydrocarbons. Extraction methods such as headspace extraction coupled to gas chromatography-mass spectrometry (GC-MS) have been used for the analysis of volatile components in food (Wu and Wang, 2000; Akiyama et al., 2005), particularly those in citrus (Steffen and Pawliszun, 1996; Jia et al., 1998; Braddock et al., 2004).

Previously, most of the studies on headspace volatile components of citrus juices had been performed on orange juice (Jordán et al., 2005; Mahattanatawee et al., 2005; Berlinet et al., 2007). In contrast to the research conducted on oranges, less information has been reported for other citrus fruits (Tamura et al., 1999; Dharmawan et al., 2007). In addition, few studies comparing volatile components among

*To whom correspondence should be addressed.

E-mail: hohta@nakamura-u.ac.jp several citrus species have been performed. Sawamura et al. (1991) classified 37 kinds of citrus fruits into 4 groups using oxygenated components. González-Mas et al. (2011) differentiated 4 citrus species from each other using volatile components. Furthermore, a study comparing chemical classification using volatile components and morphological classification, such as Tanaka's classification (Tanaka, 1969), would be useful in resolving Citrus taxonomy.

\section{Materials and Methods}

Materials The citrus fruit samples used in this study are listed according to Tanaka's classification (Tanaka, 1969) in Table 1. The fruits were purchased from the National Institute of Fruit Tree Science Okitsu Citrus Research Station (Shizuoka, Japan) in early December, 2009. Each juice was obtained by squeezing whole fruits using $260-\mathrm{mm}$-long hand-press juicers with an 84-mm diameter and 32-mm-deep pressing area.

SPME-GC, GC/MS for volatile components An aliquot $(1 \mathrm{~mL})$ of a juice sample was placed in a vial $(22 \times 38$ $\mathrm{mm}$ ) containing an internal standard solution of $10 \mu \mathrm{L}$ of $1 \%$ cyclohexanol in water. The solution was held at $40^{\circ} \mathrm{C}$ for 5 min, and a Sigma-Aldrich Carboxen / PDMS SPME 
Table 1. Classification of Citrus Plants Investigated in an Analysis of Volatile Components.

\begin{tabular}{|c|c|c|c|c|c|c|c|}
\hline $\mathrm{Cla}$ & $\begin{array}{l}\text { ification of ci } \\
\text { Tanaka's class }\end{array}$ & $\begin{array}{l}\text { us based on } \\
\text { ication }\end{array}$ & & $\#$ & $\begin{array}{c}\text { Tanaka’s } \\
\text { NO. }\end{array}$ & Scientific name & Conventional name \\
\hline \multirow[t]{39}{*}{ Citrus } & Archicitrus & Limonellus & Eulimonellus & 1 & II-4-14 & C. latifolia & Tahiti lime \\
\hline & & & & 2 & II-4-15 & C. limettioides & Sweet lime \\
\hline & & Citrophorum & Citrioides & 3 & III-7-31 & C. medica & Citron \\
\hline & & & Limonioides & 4 & III-8-36 & C. limon & Eureka lemon \\
\hline & & & & 5 & III-8-38 & C. limetta & Sweet lemon \\
\hline & & & Decumanoides & 6 & III-9-48 & C. lumia & Lumie \\
\hline & & Cephalocitrus & Decumana & 7 & IV-10-56 & C. grandis & Hirado buntan \\
\hline & & & & 8 & IV-10-61 & C. grandis & Shaten yu \\
\hline & & & Intermedia & & & & \\
\hline & & & Flavicarpa & 9 & IV-11-62 & C. paradisi & Marsh grapefruit \\
\hline & & & & 10 & IV-11-63 & C. glaberrima & Kinukawa \\
\hline & & & Aureocarpa & 11 & IV-12-74 & C. hassaku & Hassaku \\
\hline & & Aurantium & $\begin{array}{l}\text { Medioglobosa } \\
\text { Aurantioides }\end{array}$ & 12 & V-13-78 & C. natsudaidai & Natsudaidai \\
\hline & & & Racemosa & 13 & V-14-93 & C. aurantium & Sour orange \\
\hline & & & Sinensoides & 14 & V-16-100 & C. sinensis & Valencia \\
\hline & & & & 15 & V-16-100 & C. sinensis & Navel \\
\hline & & & & 16 & V-16-105 & C. iyo & Iyo \\
\hline & & & Osmocitroides & & & & \\
\hline & & & Tenuicarpa & 17 & V-17-107 & C. tamurana & Hyuganatsu \\
\hline & & & Paranobilis & 18 & V-19-111 & C. shunkokan & Shunkokan \\
\hline & Metacitrus & Osmocitrus & Euosmocitroides & 19 & VI-21-113 & C. junos & Yuzu \\
\hline & & & & 20 & VI-21-121 & C. sphaerocarpa & Kabosu \\
\hline & & Acrumen & Euacrumen & 21 & VII-23-123 & C. nobilis & King \\
\hline & & & & 22 & VII-23-124 & C. unshiu & Unshu \\
\hline & & & & 23 & VII-23-125 & C. yatsushiro & Yatsushiro \\
\hline & & & Microacrumen & & & & \\
\hline & & & Anisodora & 24 & VII-24-126 & C. keraji & Keraji \\
\hline & & & & 25 & VII-24-127 & C. oto & Oto \\
\hline & & & Citroidora & & & & \\
\hline & & & Megacarpa & 26 & VII-25-130 & C. reticulata & Ponkan \\
\hline & & & & 27 & VII-25-133 & C. tangerina & Dancy Tangerine \\
\hline & & & & 28 & VII-25-134 & C. clementia & Clementine \\
\hline & & & & 29 & VII-25-136 & C. succosa & Jimikan \\
\hline & & & Microcarpa & 30 & VII-26-143 & C. tachibana & Tachibana \\
\hline & & & & 31 & VII-26-145 & C. kinokuni & Kishu \\
\hline & & & & 32 & VII-27-148 & C. sunki & Sunki \\
\hline & & & & 33 & VII-27-153 & C. depressa & Shiikuwasha \\
\hline & & & & 34 & VII-27-154 & C. leiocarpa & Koji \\
\hline & & Pseudofortunella & & 35 & VIII-28-159 & C. madurensis & Shikikitsu \\
\hline \multirow[t]{2}{*}{ Fortunella } & & & & 36 & & F. margarita & Nagami kumquat \\
\hline & & & & 37 & & F. crassifolia & Ninpou kumquat \\
\hline
\end{tabular}

fiber (fiber length of $10 \mathrm{~mm}, 75 \mu \mathrm{m}$ of film thickness) was introduced into the septum-sealed vial and kept in the injection port for $20 \mathrm{~min}$. SPME cryofocusing was carried out as follows. Heating of the GC column-oven was stopped and a column head $(10 \mathrm{~cm})$ of the $\mathrm{GC}$ was dipped into liquid nitrogen to collect the volatiles in splitless mode. Then, the SPME fiber was introduced into the injector and kept there for 7 min (Hashimoto et al., 2006). Volatile components of the headspace gas were analyzed using a Shimadzu GC14A gas chromatograph. A $0.25 \mathrm{~mm}$ i.d. $\times 60 \mathrm{~m}$ DB-WAX chemical bond type silica capillary column with $0.25-\mu \mathrm{m}-$ thick film (Agilent Technologies, Santa Clara, CA, U.S.A.) was employed, with the FID at $230^{\circ} \mathrm{C}$ and injection port at $260^{\circ} \mathrm{C}$. The temperature was programmed from $40^{\circ} \mathrm{C}$ to 
$230^{\circ} \mathrm{C}$ at $3^{\circ} \mathrm{C} / \mathrm{min}$, and to be held at that temperature for 10 min. The helium carrier gas flow through the column was 1.0 $\mathrm{mL} / \mathrm{min}$. The headspace gas constituents were identified by gas chromatography-mass spectrometry. A Varian 3400 gas chromatograph interfaced to a Finigan MAT Model 800 ion trap detector (Thermo Fisher Scientific, Waltham, MA, USA) was used. Transfer port temperature and ion trap temperature were kept at $230^{\circ} \mathrm{C}$. Mass units were monitored from $26-$ $300 \mathrm{aum} / \mathrm{s}$ at $1250 \mathrm{eV}$. Other conditions were the same as those of GC conditions. The components were identified by comparing the mass spectra of eluted compounds with that in a library search system (MAGNUM Library-Search System: NIST MASS SPECTRA DATABASE 62,235 compounds) and their GC retention indices (RI) on a DB-WAX column with those of authentic compounds (Wako Pure Chemical Industries, Osaka, Japan; Tokyo Kasei Kogyo, Tokyo, Japan; Sigma-Aldrich, St. Louis, MO, USA) previously analyzed and stored in our private data file. The GC data was calculated using a Shimadzu GC-6R integrator. All treatments were performed in duplicate and the results are presented as mean values.

Statistical analysis Principal component analysis (PCA) and cluster analysis were performed using the SPSS statistics version 19.0. (IBM Corp., Armonk, NY, USA). The peak area percentages (\%) of 48 volatile compounds of the citrus juice were used as variables for principal component analysis. Subsequently, the principal component scores calculated by PCA were used for the cluster analysis. Dendrograms were generated using the Ward method, which uses squared Euclidean distance with z-score normalized variables.

\section{Results and Discussion}

Composition of volatile compounds from various citrus juices All the citrus fruits assessed in this study were cultivated under the same weather conditions to reduce factors of variance; samples were collected from the same fields and in the same year. We divided 37 citrus samples into 18 Archicitrus, 17 Metacitrus and 2 Fortunella species based on Tanaka's classification (Tanaka, 1969), as the morphological characteristics differed vastly among Archicitrus, Metacitrus and Fortunella. Because Pseudofortunella resembles Fortunella in flavor, we decided to compare Metacitrus and Fortunella. Table 2 shows the volatile compositions in 18 Archicitrus citrus juices, and Table 3 summarizes those in 17 Metacitrus and 2 Fortunella citrus juices. The compounds are listed in a classification that is based on the functional group. Forty-eight compounds from more than 80 peaks on gas chromatograms were identified and quantified.

As shown in Table 2, hydrocarbons were dominant in the headspace gas of Archicitrus juice, followed by alcohols, aldehydes, ketones, esters and acids. Limonene was the most abundant hydrocarbon in the Archicitrus juice samples tested in this study, and the second most abundant hydrocarbon was $\gamma$-terpinene or myrcene. Limonene was the main constituent of all samples in the Archicitrus. Hirado buntan (\#7) and Marsh grapefruit (\#9) contained the highest percentage of limonene in the Archicitrus. Sawamura et al. (1990) reported that Hirado buntan contained the highest percentage of limonene $(86.31 \%)$, followed by myrcene $(2.46 \%)$. Attaway et al. (1967) showed that in grapefruit the highest percentage was limonene $(93 \%)$, followed by myrcene $(1.9 \%)$. Our results generally agree with their data, except for minor compounds. A high percentage of $\gamma$-terpinene was present in Tahiti lime (\#1) and Citron (\#3). Of the alcohols, linalool was the most abundant component, followed by 1-octanol. Lumie (\#6) contained exceptionally large amounts of linalool and 1-octanol. Limonellus (II) samples contained a low percentage of oxygenated compounds, except for alcohols. Lumie (\#6) contained the highest percentage of oxygenated compounds in the Archicitrus; however, intermediate percentages of these were detected in other Citrophorum (III) citrus. In Archicitrus citrus, Cephalocitrus (IV) samples, except Kinukawa (\#10), contained higher percentages of hydrocarbons. Aurantium (V) samples contained low percentages of oxygenated compounds, except for aldehydes; however, Iyo (\#16) contained a small amount of aldehydes.

As shown in Table 3, the most abundant component in the headspace gas of Metacitrus citrus juices was hydrocarbons, followed by alcohols, aldehydes, ketones, esters and acids. This composition of volatile components was quite similar to Archicitrus. Similarly, dominant components such as limonene, $\gamma$-terpinene, myrcene and linalool closely resemble each other. Yatsushiro (\#23), Oto (\#25) and Shiikuwasha (\#33) contained the highest percentage of $\gamma$-terpinene. Inafuku et al. (2011) revealed $C$. depressa contained the highest percentage of $\gamma$-terpinene, except for limonene. Kabosu (\#20) and Keraji (\#24) contained a high percentage of myrcene, and Keraji contained the highest percentage of citronellol. In the Osmocitrus (VI) samples and Fortunella, high percentages of hydrocarbons were present; however, only a low percentage of oxygenated compounds was detected. Akakabe et al. (2008) compared the Japanese sour citruses in Osmocitrus, and detected a high percentage of hydrocarbons. Furthermore, Choi (2005) showed kumquat contained a high percentage of hydrocarbons; our results agree with their data. In the Acrumen (VII) samples, it was too difficult to identify distinguishing characteristics, because 1 citrus sample showed several characteristics. Since the 13 Acrumen samples exhibited highly variable characteristics, it was difficult to generalize about their characteristics. 
Table 2-1. Composition of volatile components in the headspace gas of Citrus Archicitrus species.

\begin{tabular}{|c|c|c|c|c|c|c|c|c|c|c|}
\hline \# & & 1 & 2 & 3 & 4 & 5 & 6 & 7 & 8 & 9 \\
\hline Tanaka's No. & & II-4-14 & II-4-15 & III-7-31 & III-8-36 & III-8-38 & III-9-48 & IV-10-56 & IV-10-61 & IV-11-62 \\
\hline Compound & ${ }^{*} \mathrm{RI}$ & Tahiti lime & Sweet lime & Citron & $\begin{array}{l}\text { Eureka } \\
\text { lemon }\end{array}$ & $\begin{array}{l}\text { Sweet } \\
\text { lemon }\end{array}$ & Lumie & $\begin{array}{l}\text { Hirado } \\
\text { buntan }\end{array}$ & Shaten yu & $\begin{array}{c}\text { Marsh } \\
\text { grapefruit }\end{array}$ \\
\hline \multicolumn{11}{|l|}{ Hydrocarbons } \\
\hline$\alpha$-Pinene & 1025 & 1.83 & 0.10 & 0.53 & 1.23 & 0.05 & 0.34 & 0.12 & 0.10 & 0.15 \\
\hline$\alpha$-Thujene & 1029 & 0.38 & nd & 0.29 & 0.22 & nd & 0.10 & nd & 0.01 & 0.01 \\
\hline Toluene & 1037 & $\operatorname{tr}$ & 0.01 & 0.01 & 0.01 & 0.02 & 0.01 & 0.01 & 0.03 & nd \\
\hline$\beta$-Pinene & 1114 & 10.50 & nd & 0.90 & 8.36 & 0.03 & 5.16 & 0.59 & 0.04 & 0.02 \\
\hline Myrcene & 1168 & 2.10 & 3.27 & 2.48 & 3.10 & 3.21 & 3.71 & 2.37 & 3.47 & 1.93 \\
\hline$\alpha$-Terpinene & 1182 & 0.54 & nd & 0.78 & 0.35 & 0.06 & 0.02 & nd & nd & nd \\
\hline Limonene & 1205 & 49.27 & 88.23 & 49.23 & 66.69 & 88.43 & 64.16 & 90.16 & 88.70 & 90.25 \\
\hline$(Z)$ - $\beta$-Ocymene & 1240 & 0.06 & 0.02 & 0.36 & 0.06 & 0.02 & 0.31 & nd & 0.02 & 0.02 \\
\hline$\gamma$-Terpinene & 1255 & 18.90 & nd & 23.47 & 9.28 & 0.34 & 1.15 & nd & 0.47 & 0.07 \\
\hline (E)-b-Ocymene & 1256 & nd & nd & nd & nd & nd & nd & nd & nd & nd \\
\hline p-Cymene & 1277 & 1.31 & 0.38 & 8.29 & 1.24 & 0.92 & 0.01 & 0.68 & 0.56 & 0.18 \\
\hline Terpinolene & 1291 & 1.04 & 0.02 & 1.56 & 0.68 & 0.05 & 0.01 & 0.13 & 0.07 & 0.08 \\
\hline$\delta$-Elemene & 1478 & nd & 0.03 & nd & nd & 0.02 & nd & 0.08 & 0.10 & nd \\
\hline$\alpha$-Copaene & 1502 & 0.03 & nd & 0.22 & 0.02 & nd & nd & nd & 0.01 & 0.10 \\
\hline$\beta$-Caryophyllene & 1616 & nd & nd & nd & 0.37 & nd & 0.17 & nd & nd & 0.75 \\
\hline$\alpha$-Caryophyllene & 1685 & 0.06 & 0.05 & 0.03 & 0.12 & 0.02 & 0.06 & nd & 0.02 & 0.10 \\
\hline Germacrene D & 1724 & nd & 0.13 & nd & nd & nd & nd & nd & nd & 0.09 \\
\hline$\beta$-Bisabolene & 1748 & nd & 0.05 & 0.65 & 1.33 & nd & 0.50 & nd & 0.56 & 0.20 \\
\hline \multicolumn{11}{|l|}{ Acids } \\
\hline Acetic acid & 1449 & nd & nd & nd & 0.01 & nd & nd & 0.03 & nd & nd \\
\hline \multicolumn{11}{|l|}{ Alcohols } \\
\hline Ethanol & 933 & 1.05 & 0.88 & 0.04 & 0.19 & 0.06 & 0.02 & 0.09 & 0.31 & nd \\
\hline 1-Penten-3-ol & 1159 & nd & nd & nd & 0.01 & nd & 0.01 & nd & nd & nd \\
\hline Cineol & 1214 & 0.26 & 0.04 & 0.11 & nd & nd & 0.01 & nd & 0.14 & nd \\
\hline 1-Heptanol & 1458 & 0.04 & 0.03 & 0.03 & 0.04 & 0.04 & 0.04 & 0.01 & 0.01 & 0.03 \\
\hline Linalool & 1550 & 0.17 & 1.20 & 0.34 & 0.13 & 0.80 & 12.24 & 0.09 & 0.57 & 0.10 \\
\hline 1-Octanol & 1560 & 0.04 & 0.06 & 0.34 & 0.04 & 0.04 & 5.53 & 0.04 & 0.02 & 0.11 \\
\hline Terpinene-4-ol & 1608 & 1.18 & 0.02 & 0.63 & 0.99 & 0.02 & 0.05 & 0.14 & 0.02 & 0.19 \\
\hline $\begin{array}{l}p \text {-Mentha- } \\
(E) \text {-2,8-dien-1-ol }\end{array}$ & 1633 & nd & nd & 0.05 & nd & 0.03 & nd & nd & 0.02 & 0.09 \\
\hline$\alpha$-Terpineol & 1702 & 1.10 & 0.05 & 1.68 & 0.31 & 0.06 & 0.19 & 0.11 & 0.22 & 0.05 \\
\hline Citronellol & 1768 & 0.88 & 1.33 & 0.26 & 0.52 & nd & 1.07 & 0.25 & 0.21 & 0.36 \\
\hline$p$-Cymene-8-ol & 1852 & 0.01 & nd & nd & nd & nd & 0.14 & nd & nd & nd \\
\hline$\beta$-Elemol & 2086 & nd & nd & nd & 0.01 & nd & nd & nd & 0.01 & 0.01 \\
\hline$\tau$-Cadinol & 2180 & nd & nd & nd & nd & nd & 0.01 & nd & nd & nd \\
\hline Thymol & 2185 & 0.02 & nd & 0.16 & 0.01 & 0.03 & 0.01 & 0.01 & 0.08 & 0.03 \\
\hline Iso thymol & 2215 & nd & nd & 0.34 & 0.01 & nd & $\operatorname{tr}$ & nd & 0.01 & nd \\
\hline \multicolumn{11}{|l|}{ Aldehydes } \\
\hline Hexanal & 1081 & nd & 0.10 & nd & 0.02 & 0.18 & 0.01 & nd & 0.10 & nd \\
\hline Heptanal & 1185 & nd & 0.01 & nd & nd & nd & nd & nd & 0.01 & nd \\
\hline Octanal & 1290 & 0.07 & 0.02 & 0.16 & 0.12 & 0.10 & 0.05 & 0.03 & 0.02 & 1.30 \\
\hline Nonanal & 1395 & 0.06 & 0.04 & 0.45 & 0.45 & nd & nd & nd & 0.11 & 0.20 \\
\hline Decanal & 1501 & 0.29 & nd & 0.47 & 0.09 & 0.31 & 0.23 & 0.17 & 0.47 & 1.00 \\
\hline Myrtenal & 1638 & 0.05 & nd & nd & nd & nd & 0.03 & 0.01 & nd & nd \\
\hline (E)-2-Decenal & 1647 & 0.02 & nd & nd & 0.04 & nd & nd & nd & nd & 0.01 \\
\hline \multicolumn{11}{|l|}{ Esters } \\
\hline Ethyl acetate & 886 & 0.01 & 0.03 & 0.02 & 0.01 & 0.02 & 0.01 & 0.01 & 0.02 & nd \\
\hline Heptyl acetate & 1376 & nd & nd & nd & nd & nd & nd & nd & nd & nd \\
\hline Octyl acetate & 1477 & $\operatorname{tr}$ & nd & 0.11 & 0.09 & nd & nd & nd & 0.02 & 0.09 \\
\hline Linalyl acetate & 1554 & nd & 0.02 & 0.06 & 0.01 & 0.02 & nd & nd & 0.03 & 0.01 \\
\hline Perillyl acetate & 1862 & 0.03 & nd & 0.02 & 0.12 & nd & 0.01 & 0.05 & 0.04 & 0.02 \\
\hline \multicolumn{11}{|l|}{ Ketones } \\
\hline Camphor & 1534 & nd & nd & nd & 0.01 & nd & nd & nd & nd & nd \\
\hline D-Carvone & 1742 & nd & nd & 0.27 & 0.79 & 0.06 & 1.36 & nd & 0.06 & nd \\
\hline Hydrocarbons & & 86.01 & 92.28 & 88.81 & 93.05 & 93.16 & 75.71 & 94.14 & 94.15 & 93.95 \\
\hline Acids & & 0.00 & 0.00 & 0.00 & 0.01 & 0.00 & 0.00 & 0.03 & 0.00 & 0.00 \\
\hline Alcohols & & 4.76 & 3.60 & 3.98 & 2.24 & 1.09 & 19.32 & 0.74 & 1.63 & 0.96 \\
\hline Aldehydes & & 0.49 & 0.18 & 1.09 & 0.72 & 0.59 & 0.32 & 0.21 & 0.72 & 2.50 \\
\hline Esters & & 0.04 & 0.05 & 0.21 & 0.23 & 0.04 & 0.02 & 0.06 & 0.10 & 0.12 \\
\hline Ketones & & 0.00 & 0.00 & 0.27 & 0.79 & 0.06 & 1.36 & 0.00 & 0.06 & 0.00 \\
\hline Total & & 91.29 & 96.11 & 94.36 & 97.04 & 94.92 & 96.73 & 95.17 & 96.67 & 97.54 \\
\hline
\end{tabular}

mean $(n=2)$ nd: not detected; tr: trace $(<0.005 \%)^{*}$ : RI: Retention indices 
Table 2-2. Composition of volatile components in the headspace gas of Citrus Archicitrus species (continued).

\begin{tabular}{|c|c|c|c|c|c|c|c|c|c|c|}
\hline$\#$ & & 10 & 11 & 12 & 13 & 14 & 15 & 16 & 17 & 18 \\
\hline Tanaka's No. & & IV-11-63 & IV-12-74 & V13-78 & V-14-93 & V-16-100 & V-16-100 & V-15-105 & V-17-107 & V-19-111 \\
\hline Compound & ${ }^{*} \mathrm{RI}$ & Kinukawa & Hassaku & Natsudaidai & Sour orange & Valencia & Navel & Iyo & Hyuganatsu & Shunkokan \\
\hline \multicolumn{11}{|l|}{ Hydrocarbons } \\
\hline$\alpha$-Pinene & 1025 & 0.11 & 0.16 & 0.23 & 0.30 & 0.25 & 0.10 & 0.31 & 0.27 & 0.14 \\
\hline$\alpha$-Thujene & 1029 & 0.04 & 0.04 & 0.05 & 0.04 & 0.04 & nd & 0.09 & 0.08 & nd \\
\hline Toluene & 1037 & nd & nd & 0.01 & nd & 0.01 & 0.22 & 0.01 & 0.01 & 0.02 \\
\hline$\beta$-Pinene & 1114 & 0.17 & 0.20 & 0.18 & 0.97 & 0.03 & 0.02 & 0.30 & 0.35 & 0.61 \\
\hline Myrcene & 1168 & 2.96 & 3.39 & 2.64 & 2.79 & 3.16 & 2.34 & 2.49 & 1.58 & 3.09 \\
\hline$\alpha$-Terpinene & 1182 & 0.22 & 0.17 & 0.06 & nd & 0.14 & 0.05 & 0.05 & 0.16 & 0.09 \\
\hline Limonene & 1205 & 73.17 & 81.14 & 84.23 & 88.84 & 89.45 & 86.85 & 82.60 & 77.48 & 85.71 \\
\hline$(Z)$ - $\beta$-Ocymene & 1240 & 0.05 & 0.02 & 0.02 & nd & nd & 0.02 & $\operatorname{tr}$ & 0.05 & 0.14 \\
\hline$\gamma$-Terpinene & 1255 & 4.65 & 6.61 & 5.06 & 1.09 & nd & 0.26 & 0.79 & 8.36 & 0.39 \\
\hline (E)- $\beta$-Ocymene & 1256 & 0.73 & nd & nd & nd & nd & nd & 6.65 & nd & 0.19 \\
\hline p-Cymene & 1277 & 2.17 & 2.20 & 0.92 & 0.17 & nd & 0.40 & 0.64 & 1.12 & 0.50 \\
\hline Terpinolene & 1291 & 0.47 & 0.58 & 0.38 & 0.08 & 0.09 & 0.14 & 0.48 & 0.57 & 0.14 \\
\hline$\delta$-Elemene & 1478 & 0.09 & 0.11 & 0.02 & 0.20 & nd & nd & nd & nd & nd \\
\hline$\alpha$-Copaene & 1502 & 0.03 & nd & 0.04 & $\operatorname{tr}$ & nd & 0.01 & $\operatorname{tr}$ & 0.01 & 0.04 \\
\hline$\beta$-Caryophyllene & 1616 & nd & nd & nd & 0.04 & nd & 0.01 & 0.01 & nd & 0.04 \\
\hline$\alpha$-Caryophyllene & 1685 & 0.07 & 0.05 & 0.05 & 0.07 & nd & 0.01 & 0.02 & 0.04 & 0.02 \\
\hline Germacrene D & 1724 & nd & nd & 0.04 & 0.09 & 0.05 & 0.21 & 0.03 & nd & nd \\
\hline$\beta$-Bisabolene & 1748 & 0.04 & 0.03 & 0.06 & 0.12 & 0.32 & 0.32 & nd & 0.13 & 0.18 \\
\hline \multicolumn{11}{|l|}{ Acids } \\
\hline Acetic acid & 1449 & nd & nd & nd & nd & 0.01 & nd & nd & nd & nd \\
\hline \multicolumn{11}{|l|}{ Alcohols } \\
\hline Ethanol & 933 & 1.25 & 0.02 & 0.06 & 0.01 & 0.30 & 1.05 & 0.01 & 0.25 & 0.05 \\
\hline 1-Penten-3-ol & 1159 & nd & nd & nd & nd & nd & nd & nd & nd & 0.02 \\
\hline Cineol & 1214 & 0.06 & 0.10 & 0.05 & nd & nd & 0.04 & nd & 0.06 & 0.05 \\
\hline 1-Heptanol & 1458 & 0.04 & 0.02 & 0.04 & 0.03 & nd & nd & 0.02 & 0.02 & nd \\
\hline Linalool & 1550 & 4.86 & 0.18 & 0.13 & 0.25 & 0.15 & 0.48 & 1.48 & 3.19 & 0.91 \\
\hline 1-Octanol & 1560 & 1.34 & 0.63 & 0.42 & 0.04 & 0.12 & 1.09 & 0.07 & 0.03 & 0.26 \\
\hline Terpinene-4-ol & 1608 & 0.15 & 0.08 & 0.12 & 0.13 & 0.18 & 0.58 & 0.13 & 0.38 & 0.38 \\
\hline $\begin{array}{l}p \text {-Mentha- } \\
(E) \text {-2,8-dien-1-ol }\end{array}$ & 1633 & nd & nd & 0.02 & 0.01 & nd & nd & 0.01 & 0.04 & nd \\
\hline$\alpha$-Terpineol & 1702 & 0.19 & 0.19 & 0.12 & 0.12 & 0.09 & 0.11 & 0.10 & 0.32 & 0.54 \\
\hline Citronellol & 1768 & 0.49 & 0.27 & 0.29 & 0.45 & nd & 0.22 & 0.09 & 0.05 & 0.03 \\
\hline p-Cymene-8-ol & 1852 & 0.02 & nd & nd & nd & nd & nd & 0.01 & 0.02 & 0.03 \\
\hline$\beta$-Elemol & 2086 & 0.04 & nd & 0.02 & $\operatorname{tr}$ & nd & nd & nd & nd & nd \\
\hline$\tau$-Cadinol & 2180 & nd & nd & nd & nd & nd & nd & 0.01 & nd & nd \\
\hline Thymol & 2185 & 0.03 & nd & 0.01 & 0.01 & 0.01 & 0.01 & 0.01 & 0.01 & 0.04 \\
\hline Iso thymol & 2215 & nd & nd & nd & nd & 0.01 & 0.01 & $\operatorname{tr}$ & nd & nd \\
\hline \multicolumn{11}{|l|}{ Aldehydes } \\
\hline Hexanal & 1081 & 0.39 & 0.21 & 0.02 & nd & 0.01 & 0.02 & 0.02 & 0.23 & 0.12 \\
\hline Heptanal & 1185 & nd & 0.09 & 0.01 & nd & nd & 0.03 & nd & 0.04 & nd \\
\hline Octanal & 1290 & 0.04 & 0.05 & 0.72 & 1.09 & nd & 1.27 & 0.16 & 0.37 & 0.02 \\
\hline Nonanal & 1395 & 0.19 & 0.13 & 0.23 & 0.11 & nd & 0.13 & 0.09 & 0.15 & 0.73 \\
\hline Decanal & 1501 & 0.20 & 0.19 & 0.81 & 0.53 & 0.90 & 0.85 & 0.25 & 0.15 & 0.29 \\
\hline Myrtenal & 1638 & nd & nd & nd & nd & nd & nd & nd & nd & nd \\
\hline$(E)$-2-Decenal & 1647 & nd & nd & nd & 0.04 & nd & 0.01 & 0.02 & nd & nd \\
\hline \multicolumn{11}{|l|}{ Esters } \\
\hline Ethyl acetate & 886 & nd & nd & nd & $\operatorname{tr}$ & $\operatorname{tr}$ & 0.01 & $\operatorname{tr}$ & 0.03 & 0.19 \\
\hline Heptyl acetate & 1376 & nd & nd & 0.01 & 0.01 & nd & nd & nd & nd & 0.03 \\
\hline Octyl acetate & 1477 & 0.15 & 0.08 & 0.24 & 0.13 & nd & 0.03 & 0.03 & 0.04 & 0.02 \\
\hline Linalyl acetate & 1554 & 0.05 & 0.02 & 0.04 & 0.04 & nd & 0.02 & 0.03 & 0.04 & 0.02 \\
\hline Perillyl acetate & 1862 & 0.13 & 0.03 & 0.08 & $\operatorname{tr}$ & 0.01 & 0.02 & 0.04 & 0.05 & 0.10 \\
\hline \multicolumn{11}{|l|}{ Ketones } \\
\hline Camphor & 1534 & nd & nd & nd & nd & nd & nd & nd & nd & 0.09 \\
\hline D-Carvone & 1742 & 0.05 & 0.03 & 0.02 & 0.04 & nd & 0.02 & 0.03 & 0.02 & 0.06 \\
\hline Hydrocarbons & & 84.96 & 94.71 & 93.97 & 94.81 & 93.55 & 90.97 & 94.48 & 90.21 & 91.29 \\
\hline Acids & & 0.00 & 0.00 & 0.00 & 0.00 & 0.01 & 0.00 & 0.00 & 0.00 & 0.00 \\
\hline Alcohols & & 8.46 & 1.48 & 1.28 & 1.04 & 0.86 & 3.59 & 1.95 & 4.36 & 2.31 \\
\hline Aldehydes & & 0.82 & 0.66 & 1.78 & 1.78 & 0.91 & 2.31 & 0.54 & 0.95 & 1.16 \\
\hline Esters & & 0.33 & 0.14 & 0.37 & 0.18 & 0.01 & 0.07 & 0.09 & 0.16 & 0.36 \\
\hline Ketones & & 0.05 & 0.03 & 0.02 & 0.04 & 0.00 & 0.02 & 0.03 & 0.02 & 0.16 \\
\hline Total & & 94.63 & 97.01 & 97.42 & 97.84 & 95.34 & 96.95 & 97.09 & 95.71 & 95.28 \\
\hline
\end{tabular}


Table 3-1. Composition of volatile components in the headspace gas of Citrus Metacitrus and Fortunella species.

\begin{tabular}{|c|c|c|c|c|c|c|c|c|c|c|c|}
\hline \# & & 19 & 20 & 21 & 22 & 23 & 24 & 25 & 26 & 27 & 28 \\
\hline Tanaka's No. & & VI-21-113 & VI-21-121 & VII-23-123 & VII-23-124 & VII-23-125 & VII-24-126 & VII-24-127 & VII-25-130 & VII-25-133 & VII-25-134 \\
\hline Compound & ${ }^{*} \mathrm{RI}$ & Yиzu & Kabosu & King & Unshu & Yatsushiro & Keraji & Oto & Ponkan & $\begin{array}{l}\text { Dancy } \\
\text { tangerine }\end{array}$ & Clementine \\
\hline \multicolumn{12}{|l|}{ Hydrocarbons } \\
\hline$\alpha$-Pinene & 1025 & 1.04 & 0.11 & 0.32 & 0.14 & 0.40 & 0.20 & 0.59 & 0.24 & 0.19 & 0.07 \\
\hline$\alpha$-Thujene & 1029 & 0.28 & nd & nd & 0.03 & 0.08 & 0.07 & 0.19 & 0.08 & 0.03 & 0.06 \\
\hline Toluene & 1037 & 0.03 & 0.06 & $\operatorname{tr}$ & nd & 0.01 & 0.01 & 0.01 & 0.01 & nd & 0.02 \\
\hline$\beta$-Pinene & 1114 & 0.66 & 0.06 & 0.10 & 0.12 & 0.52 & 0.24 & 0.69 & 0.18 & 0.15 & nd \\
\hline Myrcene & 1168 & 2.04 & 19.60 & 1.83 & 3.45 & 3.02 & 28.07 & 2.33 & 1.95 & 3.11 & 3.25 \\
\hline$\alpha$-Terpinene & 1182 & 0.03 & 0.07 & 1.25 & nd & 0.50 & nd & 0.30 & 0.09 & nd & 0.26 \\
\hline Limonene & 1205 & 76.12 & 71.50 & 92.47 & 83.16 & 63.00 & 40.60 & 64.02 & 84.55 & 75.53 & 80.75 \\
\hline$(Z)$ - $\beta$-Ocymene & 1240 & 0.02 & 0.02 & nd & nd & 0.03 & nd & 0.03 & 0.01 & nd & 0.03 \\
\hline$\gamma$-Terpinene & 1255 & 8.83 & 2.38 & 0.21 & 3.14 & 15.68 & 6.03 & 18.09 & 4.25 & 4.24 & 0.48 \\
\hline (E)- $\beta$-Ocymene & 1256 & nd & nd & nd & nd & nd & nd & nd & nd & nd & 0.38 \\
\hline$p$-Cymene & 1277 & 1.44 & 0.93 & 0.12 & 1.65 & 3.90 & 0.77 & 1.94 & 0.96 & 1.21 & 0.75 \\
\hline Terpinolene & 1291 & 0.08 & 0.24 & 1.23 & 0.36 & 1.12 & 0.41 & 1.16 & 0.37 & 0.43 & 0.22 \\
\hline$\delta$-Elemene & 1478 & 0.23 & nd & nd & 0.06 & 0.02 & 0.03 & 0.02 & 0.06 & 0.04 & nd \\
\hline$\alpha$-Copaene & 1502 & 0.04 & nd & 0.01 & nd & 0.06 & 0.08 & 0.10 & 0.01 & nd & 0.04 \\
\hline$\beta$-Caryophyllene & 1616 & nd & nd & $\operatorname{tr}$ & nd & 0.15 & nd & 0.22 & 0.01 & nd & nd \\
\hline$\alpha$-Caryophyllene & 1685 & nd & 0.08 & 0.02 & 0.02 & 0.02 & 0.14 & 0.03 & 0.01 & 0.01 & nd \\
\hline Germacrene D & 1724 & 0.21 & nd & nd & nd & nd & 0.13 & nd & nd & nd & 0.03 \\
\hline$\beta$-Bisabolene & 1748 & 0.38 & nd & 0.04 & nd & 0.19 & 1.85 & 0.48 & 0.10 & 0.10 & 0.05 \\
\hline \multicolumn{12}{|l|}{ Acids } \\
\hline Acetic acid & 1449 & nd & 0.06 & nd & 0.01 & nd & nd & nd & nd & nd & nd \\
\hline \multicolumn{12}{|l|}{ Alcohols } \\
\hline Ethanol & 933 & 0.15 & 0.75 & 0.62 & 0.46 & 0.16 & 0.48 & 0.41 & 0.04 & 1.51 & 2.56 \\
\hline 1-Penten-3-ol & 1159 & nd & nd & nd & nd & nd & nd & nd & nd & nd & nd \\
\hline Cineol & 1214 & nd & 0.02 & nd & 0.09 & 0.06 & 0.01 & 0.05 & 0.01 & 0.05 & 0.14 \\
\hline 1-Heptanol & 1458 & 0.06 & 0.06 & $\operatorname{tr}$ & 0.02 & 0.02 & 0.12 & 0.04 & nd & nd & nd \\
\hline Linalool & 1550 & 0.80 & 0.04 & 0.12 & 0.32 & 2.29 & 1.29 & 1.55 & 0.76 & 4.75 & 3.11 \\
\hline 1-Octanol & 1560 & 0.01 & nd & 0.02 & 0.03 & 0.07 & 0.05 & 0.03 & 0.04 & 0.29 & 1.50 \\
\hline Terpinene-4-ol & 1608 & 0.41 & 0.15 & 0.06 & 0.09 & 0.58 & 0.22 & 0.45 & 0.02 & 0.24 & 1.30 \\
\hline $\begin{array}{l}p \text {-Mentha- } \\
(E) \text {-2,8-dien-1-ol }\end{array}$ & 1633 & 0.22 & nd & $\operatorname{tr}$ & nd & nd & 0.82 & nd & nd & nd & nd \\
\hline$\alpha$-Terpineol & 1702 & nd & nd & 0.04 & 0.07 & 0.48 & 0.32 & 0.52 & 0.13 & 0.25 & 0.19 \\
\hline Citronellol & 1768 & 0.06 & 0.02 & 0.06 & 0.10 & nd & 8.19 & 0.25 & 0.04 & 0.05 & 0.27 \\
\hline p-Cymene-8-ol & 1852 & nd & nd & nd & nd & 0.03 & nd & nd & nd & 0.03 & nd \\
\hline b-Elemol & 2086 & 0.01 & nd & nd & nd & 0.02 & 0.01 & 0.02 & nd & nd & nd \\
\hline$\tau$-Cadinol & 2180 & nd & nd & nd & nd & nd & nd & nd & nd & 0.03 & nd \\
\hline Thymol & 2185 & 0.20 & nd & 0.01 & 0.02 & 2.16 & 0.05 & 1.48 & 0.07 & 0.31 & nd \\
\hline Iso thymol & 2215 & 0.01 & nd & nd & nd & 0.02 & 0.01 & 0.01 & nd & nd & nd \\
\hline \multicolumn{12}{|l|}{ Aldehydes } \\
\hline Hexanal & 1081 & 0.03 & 0.05 & 0.02 & 0.33 & 0.07 & 0.04 & 0.02 & 0.03 & 0.03 & 0.25 \\
\hline Heptanal & 1185 & 0.01 & nd & 0.01 & nd & 0.03 & 0.18 & 0.19 & 0.04 & 0.21 & nd \\
\hline Octanal & 1290 & 0.51 & nd & $\operatorname{tr}$ & 0.04 & 0.42 & 0.79 & 0.48 & 1.67 & 0.03 & 0.03 \\
\hline Nonanal & 1395 & 0.10 & nd & $\operatorname{tr}$ & nd & 0.18 & 0.32 & 0.12 & 0.04 & 0.11 & 0.11 \\
\hline Decanal & 1501 & 0.12 & 0.68 & 0.32 & 0.16 & 0.29 & 0.82 & 0.32 & 0.94 & 0.62 & 0.27 \\
\hline Myrtenal & 1638 & 0.03 & nd & nd & nd & 0.02 & nd & 0.01 & 0.02 & 0.01 & nd \\
\hline$(E)$-2-Decenal & 1647 & nd & nd & 0.01 & nd & nd & 0.07 & nd & nd & 0.02 & nd \\
\hline \multicolumn{12}{|l|}{ Esters } \\
\hline Ethyl acetate & 886 & 0.01 & 0.01 & nd & 0.01 & nd & nd & nd & 0.01 & 0.02 & 0.05 \\
\hline Heptyl acetate & 1376 & 0.05 & nd & 0.03 & nd & nd & nd & nd & nd & nd & nd \\
\hline Octyl acetate & 1477 & nd & nd & 0.04 & nd & 0.09 & 0.15 & nd & 0.02 & nd & 0.04 \\
\hline Linalyl acetate & 1554 & 0.02 & nd & 0.01 & 0.01 & 0.03 & 0.03 & 0.02 & 0.01 & 0.03 & 0.03 \\
\hline Perillyl acetate & 1862 & 0.01 & nd & 0.02 & 0.02 & 0.03 & 0.02 & 0.04 & 0.01 & 0.03 & 0.03 \\
\hline \multicolumn{12}{|l|}{ Ketones } \\
\hline Camphor & 1534 & nd & nd & nd & nd & nd & nd & nd & nd & nd & nd \\
\hline D-Carvone & 1742 & 0.05 & nd & 0.03 & nd & 0.27 & nd & 0.47 & 0.02 & 0.03 & nd \\
\hline Hydrocarbons & & 91.42 & 95.04 & 97.59 & 92.12 & 88.70 & 78.61 & 90.20 & 92.87 & 85.05 & 86.38 \\
\hline Acids & & 0.00 & 0.06 & 0.00 & 0.01 & 0.00 & 0.00 & 0.00 & 0.00 & 0.00 & 0.00 \\
\hline Alcohols & & 1.92 & 1.04 & 0.92 & 1.19 & 5.89 & 11.57 & 4.79 & 1.12 & 7.50 & 9.07 \\
\hline Aldehydes & & 0.81 & 0.73 & 0.35 & 0.53 & 1.01 & 2.22 & 1.14 & 2.74 & 1.04 & 0.66 \\
\hline Esters & & 0.10 & 0.01 & 0.10 & 0.05 & 0.14 & 0.20 & 0.05 & 0.04 & 0.07 & 0.16 \\
\hline Ketones & & 0.05 & 0.00 & 0.03 & 0.00 & 0.27 & 0.00 & 0.47 & 0.02 & 0.03 & 0.00 \\
\hline Total & & 94.30 & 96.88 & 98.99 & 93.91 & 96.01 & 92.60 & 96.65 & 96.79 & 93.69 & 96.27 \\
\hline
\end{tabular}


Table 3-2. Composition of volatile components in the headspace gas of Citrus Metacitrus and Fortunella species (continued).

\begin{tabular}{|c|c|c|c|c|c|c|c|c|c|c|}
\hline$\#$ & & 29 & 30 & 31 & 32 & 33 & 34 & 35 & 36 & 37 \\
\hline Tanaka's No. & & VII-25-136 & VII-26-143 & VII-26-145 & VII-26-148 & VII-27-153 & VII-27-154 & VIII-28-159 & & \\
\hline Compound & ${ }^{*} \mathrm{RI}$ & Jimikan & Tachibana & Kishu & Sunki & Shiikuwasha & Koji & Shikikitsu & $\begin{array}{l}\text { Nagami } \\
\text { kumquat }\end{array}$ & $\begin{array}{l}\text { Ninpo } \\
\text { kinquat }\end{array}$ \\
\hline \multicolumn{11}{|l|}{ Hydrocarbons } \\
\hline$\alpha$-Pinene & 1025 & 0.26 & 0.51 & 0.34 & 0.46 & 0.61 & 0.26 & 0.15 & 0.20 & 0.09 \\
\hline$\alpha$-Thujene & 1029 & 0.06 & 0.13 & 0.09 & 0.16 & 0.19 & 0.02 & nd & nd & 0.04 \\
\hline Toluene & 1037 & 0.01 & $\operatorname{tr}$ & 0.01 & nd & 0.01 & 0.06 & 0.05 & nd & 0.01 \\
\hline$\beta$-Pinene & 1114 & 0.22 & 2.00 & 1.19 & 0.42 & 0.77 & nd & 0.39 & 0.01 & nd \\
\hline Myrcene & 1168 & 1.06 & 2.19 & 1.65 & 1.89 & 1.18 & 3.01 & 2.75 & 3.04 & 3.23 \\
\hline$\alpha$-Terpinene & 1182 & 0.02 & 0.29 & 0.18 & 0.04 & 0.13 & nd & 0.18 & nd & nd \\
\hline Limonene & 1205 & 80.40 & 65.24 & 79.86 & 78.79 & 57.46 & 62.70 & 78.47 & 92.94 & 86.78 \\
\hline$(Z)$ - $\beta$-Ocymene & 1240 & 0.02 & nd & 0.02 & $\operatorname{tr}$ & nd & nd & nd & 0.01 & nd \\
\hline$\gamma$-Terpinene & 1255 & 6.20 & 14.46 & 6.15 & 8.96 & 18.58 & 13.05 & 0.65 & 0.05 & 0.39 \\
\hline (E)- $\beta$-Ocymene & 1256 & nd & nd & nd & nd & nd & nd & nd & 0.06 & nd \\
\hline p-Cymene & 1277 & 1.01 & 1.11 & 1.73 & 3.14 & 5.13 & 7.78 & 3.84 & 0.10 & 0.24 \\
\hline Terpinolene & 1291 & 0.55 & 0.76 & 0.52 & 0.58 & 1.23 & 1.09 & 0.29 & 0.07 & 0.11 \\
\hline$\delta$-Elemene & 1478 & nd & 0.83 & 0.07 & nd & nd & 0.60 & 0.22 & 0.11 & 0.25 \\
\hline$\alpha$-Copaene & 1502 & nd & 0.11 & 0.01 & 0.04 & 0.04 & nd & nd & nd & 0.01 \\
\hline$\beta$-Caryophyllene & 1616 & nd & nd & 0.03 & 0.02 & nd & nd & nd & 0.02 & 0.03 \\
\hline$\alpha$-Caryophyllene & 1685 & 0.01 & 0.26 & 0.01 & 0.03 & 0.03 & nd & 0.14 & nd & 0.09 \\
\hline Germacrene D & 1724 & nd & 0.25 & nd & nd & 0.11 & nd & 0.36 & 0.05 & 0.06 \\
\hline$\beta$-Bisabolene & 1748 & 0.14 & 0.36 & 0.41 & 0.39 & 2.04 & nd & 0.09 & 0.05 & 0.26 \\
\hline \multicolumn{11}{|l|}{ Acids } \\
\hline Acetic acid & 1449 & nd & nd & 0.01 & nd & nd & 0.04 & 0.11 & nd & nd \\
\hline \multicolumn{11}{|l|}{ Alcohols } \\
\hline Ethanol & 933 & 1.65 & 0.24 & 0.56 & 0.03 & 0.14 & 0.30 & 0.45 & 0.08 & 0.03 \\
\hline 1-Penten-3-ol & 1159 & nd & nd & nd & 0.01 & nd & nd & 0.07 & nd & nd \\
\hline Cineol & 1214 & 0.03 & 0.05 & 0.03 & $\operatorname{tr}$ & nd & nd & 0.14 & 0.02 & 0.06 \\
\hline 1-Heptanol & 1458 & 0.01 & 0.12 & 0.02 & 0.01 & 0.02 & 0.05 & 0.10 & 0.05 & 0.04 \\
\hline Linalool & 1550 & 0.76 & 2.30 & 0.54 & 0.78 & 1.70 & 1.91 & 1.35 & 0.14 & 0.93 \\
\hline 1-Octanol & 1560 & 0.12 & 0.08 & 0.32 & 0.05 & 0.12 & 0.94 & 0.41 & 0.01 & 0.04 \\
\hline Terpinene-4-ol & 1608 & 0.24 & 0.90 & 0.26 & 0.22 & 0.62 & 0.66 & 0.31 & 0.07 & 0.15 \\
\hline $\begin{array}{l}p \text {-Mentha- } \\
(E) \text {-2,8-dien-1-ol }\end{array}$ & 1633 & nd & nd & 0.04 & 0.02 & 0.09 & nd & 0.13 & nd & nd \\
\hline$\alpha$-Terpineol & 1702 & 0.22 & 0.69 & 0.17 & 0.16 & 0.47 & 0.40 & 0.34 & 0.26 & 0.17 \\
\hline Citronellol & 1768 & 0.05 & 0.56 & 0.49 & 0.01 & nd & 0.08 & 0.51 & 0.40 & 0.05 \\
\hline p-Cymene-8-ol & 1852 & nd & 0.04 & 0.01 & 0.01 & 0.01 & nd & nd & $\operatorname{tr}$ & 0.19 \\
\hline$\beta$-Elemol & 2086 & nd & 0.05 & nd & $\operatorname{tr}$ & 0.01 & nd & 0.05 & $\operatorname{tr}$ & nd \\
\hline$\tau$-Cadinol & 2180 & nd & nd & nd & 0.01 & 0.01 & nd & nd & $\operatorname{tr}$ & 0.03 \\
\hline Thymol & 2185 & 0.01 & 0.03 & 0.26 & 0.02 & 0.12 & 0.03 & nd & 0.01 & 0.01 \\
\hline Iso thymol & 2215 & nd & 0.02 & 0.01 & $\operatorname{tr}$ & 0.03 & 0.02 & 0.07 & 0.01 & 0.01 \\
\hline \multicolumn{11}{|l|}{ Aldehydes } \\
\hline Hexanal & 1081 & 0.01 & 0.03 & 0.02 & $\operatorname{tr}$ & 0.03 & nd & 0.29 & $\operatorname{tr}$ & 0.40 \\
\hline Heptanal & 1185 & 0.02 & nd & 0.01 & 0.05 & 0.20 & nd & 0.04 & 0.05 & nd \\
\hline Octanal & 1290 & 2.74 & 0.06 & 0.05 & 0.52 & 1.39 & 0.11 & 1.31 & 0.03 & 0.08 \\
\hline Nonanal & 1395 & nd & 0.22 & 0.09 & 0.04 & 0.24 & nd & 0.63 & 0.02 & nd \\
\hline Decanal & 1501 & 0.89 & 0.07 & 0.32 & 0.63 & 1.01 & nd & 0.68 & 0.05 & 0.08 \\
\hline Myrtenal & 1638 & nd & nd & nd & 0.04 & 0.07 & nd & 0.10 & 0.01 & nd \\
\hline (E)-2-Decenal & 1647 & 0.01 & 0.07 & 0.01 & 0.01 & nd & nd & 0.09 & 0.02 & nd \\
\hline \multicolumn{11}{|l|}{ Esters } \\
\hline Ethyl acetate & 886 & 0.01 & 0.03 & 0.02 & 0.01 & 0.01 & 0.06 & 0.06 & $\operatorname{tr}$ & 0.06 \\
\hline Heptyl acetate & 1376 & nd & 0.01 & nd & 0.01 & nd & nd & nd & 0.01 & 0.03 \\
\hline Octyl acetate & 1477 & 0.02 & 0.48 & 0.05 & 0.06 & 0.09 & nd & 0.18 & nd & 0.20 \\
\hline Linalyl acetate & 1554 & 0.02 & 0.06 & 0.02 & 0.02 & 0.02 & nd & nd & 0.01 & 0.02 \\
\hline Perillyl acetate & 1862 & 0.01 & 0.04 & 0.02 & nd & 0.02 & 0.05 & nd & 0.04 & nd \\
\hline \multicolumn{11}{|l|}{ Ketones } \\
\hline Camphor & 1534 & nd & nd & nd & nd & nd & nd & 0.15 & 0.01 & nd \\
\hline D-Carvone & 1742 & 0.04 & nd & 0.12 & nd & 0.10 & 0.09 & 0.06 & 0.03 & 0.08 \\
\hline Hydrocarbons & & 89.95 & 88.49 & 92.27 & 94.94 & 87.50 & 88.57 & 87.57 & 96.71 & 91.58 \\
\hline Acids & & 0.00 & 0.00 & 0.01 & 0.00 & 0.00 & 0.04 & 0.11 & 0.00 & 0.00 \\
\hline Alcohols & & 3.08 & 5.07 & 2.72 & 1.34 & 3.34 & 4.40 & 3.93 & 1.03 & 1.72 \\
\hline Aldehydes & & 3.67 & 0.43 & 0.51 & 1.29 & 2.93 & 0.11 & 3.14 & 0.18 & 0.55 \\
\hline Esters & & 0.05 & 0.61 & 0.12 & 0.10 & 0.14 & 0.11 & 0.25 & 0.05 & 0.31 \\
\hline Ketones & & 0.04 & 0.00 & 0.12 & 0.00 & 0.10 & 0.09 & 0.21 & 0.04 & 0.08 \\
\hline Total & & 96.80 & 94.60 & 95.74 & 97.67 & 94.01 & 93.33 & 95.22 & 98.02 & 94.23 \\
\hline
\end{tabular}


Principal component analysis Principal component analysis (PCA) was performed in order to differentiate Citrus species based on the composition of volatile components. The data for the 48 volatile components was subjected to PCA.

Principal components and factor loadings for the composition of volatile components are listed in Table 4. Factor loadings indicate the relative extent of the contribution that each original variable contributes to the variance contained in each principal component. The cumulative contribution ratio for first principal component (PC1) and second principal component (PC2) were 36.76\% in Archicitrus, 37.39\% in Metacitrus. Previous studies showed that cumulative contribution ratios for $\mathrm{PC} 1$ and $\mathrm{PC} 2$ ranged from $33.5 \%$ to $41.05 \%$, in an analysis of flavonoids in various citrus samples (Kawaii et al., 1999; Kawaii et al., 2000; Nogata et al., 2006), which is in keeping with the present results.

Principal components for the volatile components in Archicitrus are listed in the left column of Table 4. The PC1 positively correlates with $\gamma$-terpinene most closely, followed by $\alpha$-terpinene, $\alpha$-thujene, $\alpha$-terpineol and terpinolene, and negatively correlates with limonene most closely. PC2 positively correlates with $p$-cymene-8-ol most closely, followed by D-carvone, linalool and 1-octanol, and negatively correlates with linalyl acetate and $p$-metha- $(E)$-2, 8-dien-1-ol most closely. It was assumed that $\mathrm{PC} 1$ represented the hydrocarbons, and PC2 represented the oxygenated compounds.

The scores for the two PCs are plotted as a scatter diagram in Fig. 1 (a) and (b). This figure shows that the distribution of Archicitrus species belonging to different classes can be observed. This figure shows that three groups according to Tanaka's classification appear to be separated, although some samples were not consistent with the classification. In the Limonellus (II) samples, the plots for Tahiti lime (\#1) and Sweet lime (\#2) were separated from each other. In the Citrophorum (III) samples, the plots for Citron (\#3) and Sweet lemon (\#5) are located in the fourth quadrant and in the Aurantium (V) group, respectively. In addition, they were

Table 4. Principal components and factor loadings for the composition of volatile components.

\begin{tabular}{|c|c|c|c|c|c|c|c|c|c|}
\hline & \multicolumn{2}{|c|}{ Archicitrus } & \multicolumn{2}{|c|}{$\begin{array}{l}\text { Metacitrus and } \\
\text { Fortunella }\end{array}$} & & \multicolumn{2}{|c|}{ Archicitrus } & \multicolumn{2}{|c|}{$\begin{array}{l}\text { Metacitrus and } \\
\text { Fortunella }\end{array}$} \\
\hline & 1 & 2 & 1 & 2 & & 1 & 2 & 1 & 2 \\
\hline Eigenvalue & 10.96 & 6.68 & 9.87 & 8.08 & Factor loadings & & & & \\
\hline Contribution \% & 22.84 & 13.92 & 20.56 & 16.83 & Alcohols & & & & \\
\hline Cumulative \% & 22.84 & 36.76 & 20.56 & 37.39 & Linalool & 0.03 & 0.74 & 0.23 & 0.20 \\
\hline Factor loadings & & & & & 1-Octanol & 0.04 & 0.73 & 0.01 & -0.26 \\
\hline Hydrocarbons & & & & & Terpinene-4-ol & 0.73 & 0.05 & 0.33 & 0.25 \\
\hline$\alpha$-Pinene & 0.72 & 0.31 & 0.17 & 0.63 & $p$-Mentha- $(E)$-2,8-dien-1-ol & 0.20 & -0.50 & 0.40 & 0.09 \\
\hline$\alpha$-Thujene & 0.92 & 0.16 & 0.16 & 0.64 & $\alpha$-Terpineol & 0.92 & -0.19 & 0.67 & 0.50 \\
\hline Toluene & -0.18 & -0.15 & 0.20 & -0.42 & Citronellol & 0.21 & 0.59 & 0.35 & 0.10 \\
\hline$\beta$-Pinene & 0.61 & 0.59 & 0.55 & 0.48 & $p$-Cymene-8-ol & 0.09 & 0.81 & 0.01 & -0.12 \\
\hline Myrcene & -0.20 & 0.42 & 0.16 & -0.05 & $\beta$-Elemol & -0.03 & -0.15 & 0.89 & 0.02 \\
\hline$\alpha$-Terpinene & 0.94 & -0.19 & -0.06 & 0.17 & $\tau$-Cadinol & -0.06 & 0.53 & -0.16 & -0.16 \\
\hline Limonene & -0.94 & -0.26 & -0.55 & -0.52 & Thymol & 0.60 & -0.43 & 0.05 & 0.62 \\
\hline (Z)- $\beta$-Ocymene & 0.67 & 0.24 & -0.27 & 0.38 & Iso thymol & 0.70 & -0.41 & 0.83 & -0.26 \\
\hline$\gamma$-Terpinene & 0.95 & -0.17 & 0.34 & 0.82 & Aldehydes & & & & \\
\hline (E)- $\beta$-Ocymene & -0.11 & 0.05 & -0.17 & -0.18 & Hexanal & -0.15 & -0.11 & 0.10 & -0.59 \\
\hline$p$-Cymene & 0.76 & -0.44 & 0.32 & 0.13 & Heptanal & -0.15 & -0.17 & 0.18 & 0.44 \\
\hline Terpinolene & 0.90 & -0.26 & 0.12 & 0.62 & Octanal & -0.23 & -0.36 & 0.14 & -0.02 \\
\hline$\delta$-Elemene & -0.30 & -0.17 & 0.51 & 0.00 & Nonanal & 0.41 & -0.27 & 0.88 & -0.16 \\
\hline$\alpha$-Copaene & 0.69 & -0.49 & 0.51 & 0.72 & Decanal & -0.18 & -0.42 & 0.09 & -0.03 \\
\hline$\beta$-Caryophyllene & 0.03 & 0.07 & 0.01 & 0.61 & Myrtenal & 0.44 & 0.61 & 0.56 & -0.23 \\
\hline$\alpha$-Caryophyllene & 0.33 & 0.20 & 0.79 & -0.08 & (E)-2-Decenal & 0.16 & 0.12 & 0.83 & -0.26 \\
\hline Germacrene D & -0.41 & -0.21 & 0.85 & -0.26 & Esters & & & & \\
\hline$\beta$-Bisabolene & 0.43 & 0.16 & 0.39 & 0.43 & Ethyl acetate & -0.02 & 0.00 & 0.35 & -0.57 \\
\hline Acids & & & & & Heptyl acetate & -0.09 & -0.07 & -0.15 & -0.02 \\
\hline Acetic acid & -0.19 & 0.04 & 0.45 & -0.66 & Octyl acetate & 0.18 & -0.42 & 0.73 & 0.03 \\
\hline Alcohols & & & & & Linalyl acetate & 0.28 & -0.58 & 0.29 & 0.49 \\
\hline Ethanol & 0.08 & 0.05 & -0.24 & -0.21 & Perillyl acetate & 0.17 & -0.01 & 0.02 & 0.46 \\
\hline 1-Penten-3-ol & 0.09 & 0.44 & 0.61 & -0.59 & Ketones & & & & \\
\hline Cineol & 0.58 & -0.05 & 0.31 & -0.45 & Camphor & -0.02 & 0.02 & 0.61 & -0.61 \\
\hline 1-Heptanol & 0.36 & 0.26 & 0.81 & -0.07 & D-Carvone & 0.30 & 0.75 & 0.09 & 0.59 \\
\hline
\end{tabular}


(a)

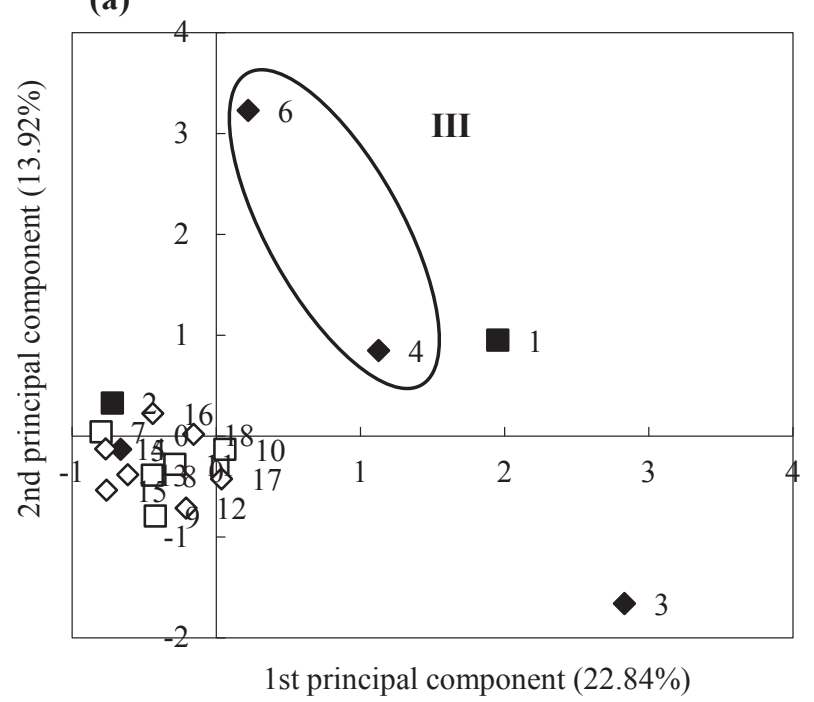

(b)

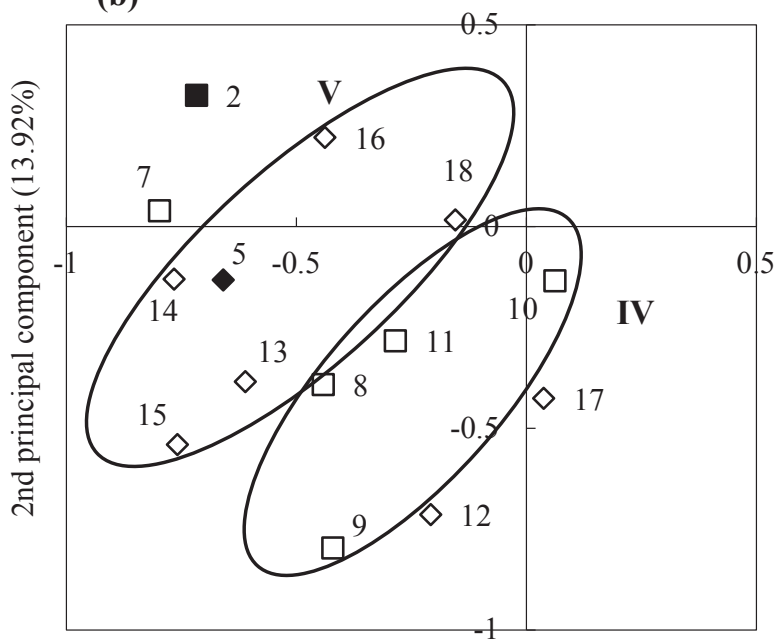

1st principal component $(22.84 \%)$

Fig. 1. Scatter diagram of principal component analysis based on the composition of volatile components in citrus juice (a) and a detail of the plot (b) .

Symbols: $\square$, Limonellus (II); $\diamond$, Citrophorum (III); $\square$, Cephacitrus (IV); $\diamond$, Aurantium (V)

Roman numerals indicate Tanaka's category number. See Table 1 for the identification of numbers.

(a)

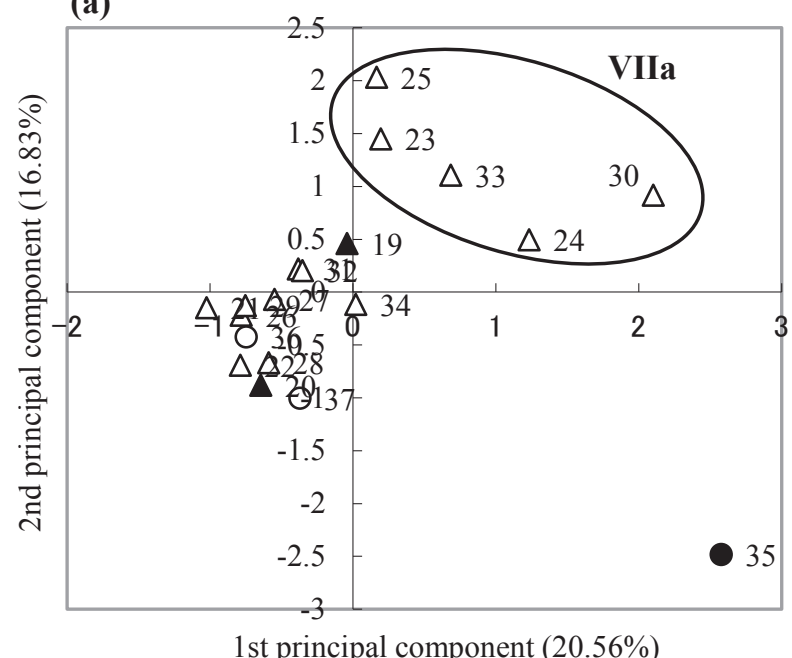

(b)

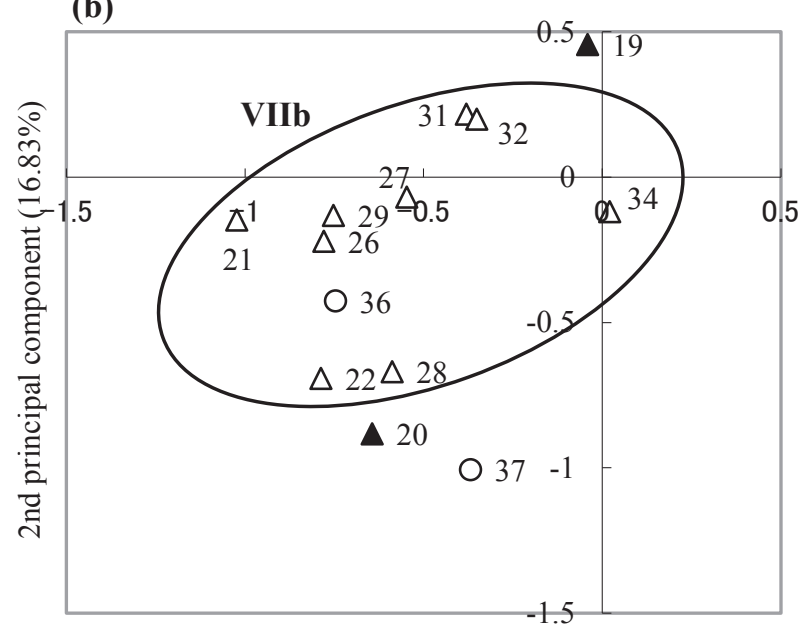

1 st principal component $(20.56 \%)$

Fig. 2. Scatter diagram of principal component analysis based on the composition of volatile components in citrus juice (a) and a detail of the plot (b) .

Symbols: $\Delta$, Osmocitrus (VI); $\triangle$, Acrumen (VII);

; Pseudofortunella (VIII); ○, Fortunella.

Roman numerals indicate Tanaka's category number. See Table 1 for the identification of numbers.

separated from those of Eureka lemon (\#4) and Lumie (\#6). Lota et al. (2002) performed PCA on volatile components in the peels of lemons and limes, and showed three major chemotypes in lemon peel oils and four chemotypes in lime peel oils. It was assumed that the Limonellus (II) and the Citrophorum (III) did not separate cleanly. Species of the Cephalocitrus (IV) formed a group separate from the others, except for Hirado buntan (\#7). In the Aurantium (V) samples, the plots for Natsudaidai (\#12) and Hyuganatsu (\#17) are close to each other, and those of others in this section formed a group within Sweet lemon (\#5).
Principal components for the volatile components in Metacitrus and Fortunella are listed in the right column of Table 4 . The PC1 positively correlates with $\beta$-elemol most closely, followed by nonanal, germacren $\mathrm{D}$, isothymol, $(E)$ 2-decanal and 1-heptanol, and negatively correlates with limonene most closely. The PC2 positively correlates with $\gamma$-terpinene and $\alpha$-copaene closely, and negatively correlates with acetic acid and camphor closely. It was assumed that PC1 represents the oxygenated compounds, and PC2 represents the hydrocarbons.

The scores for the two PCs are plotted as a scatter dia- 
gram in Fig. 2 (a) and (b). The plot for Shikikitsu (\#35) is apart from other groups. In the Osmocitrus (VI) samples, the plots for Yuzu (\#19) and Kabosu (\#20) are located in the second quadrant and the third quadrant, respectively. Akakabe et al. (2008) compared the Japanese sour citruses in Osmocitrus, and showed that the volatile composition of Yuzu differed from that of Kabosu. Interestingly, samples belonging to group VII could be divided into 2 different subgroups: VIIa and VIIb. Subgroup VIIa consists of 5 species of the VII group (i.e., Yatsushiro (\#23), Keraji (\#24), Oto (\#25), Tachibana (\#30) and Shiikuwasha (\#33)), whereas subgroup VIIb contained 8 species (i.e., Unshu (\#22), Ponkan (\#26), Dancy tangerine (\#27), Clementine (\#28), Jimikan (\#29), Kishu (\#31), Sunki (\#32) and Koji (\#34)). Shikikitsu (\#35) which belongs to the Pseudofortunella (VIII) section has a characteristic kumquat-like flavor. However, the plots of $\mathrm{Na}$ gami kumquat (\#36) and Ninpo kumquat (\#37) belong to the Fortunella genus, and that of Shikikitsu (\#35) were located far apart.

Sawamura et al. (1991) classified 37 kinds of citrus fruits into 4 groups using oxygenated components. The classification data differed in general from our result because we analyzed by PCA using different variables from those

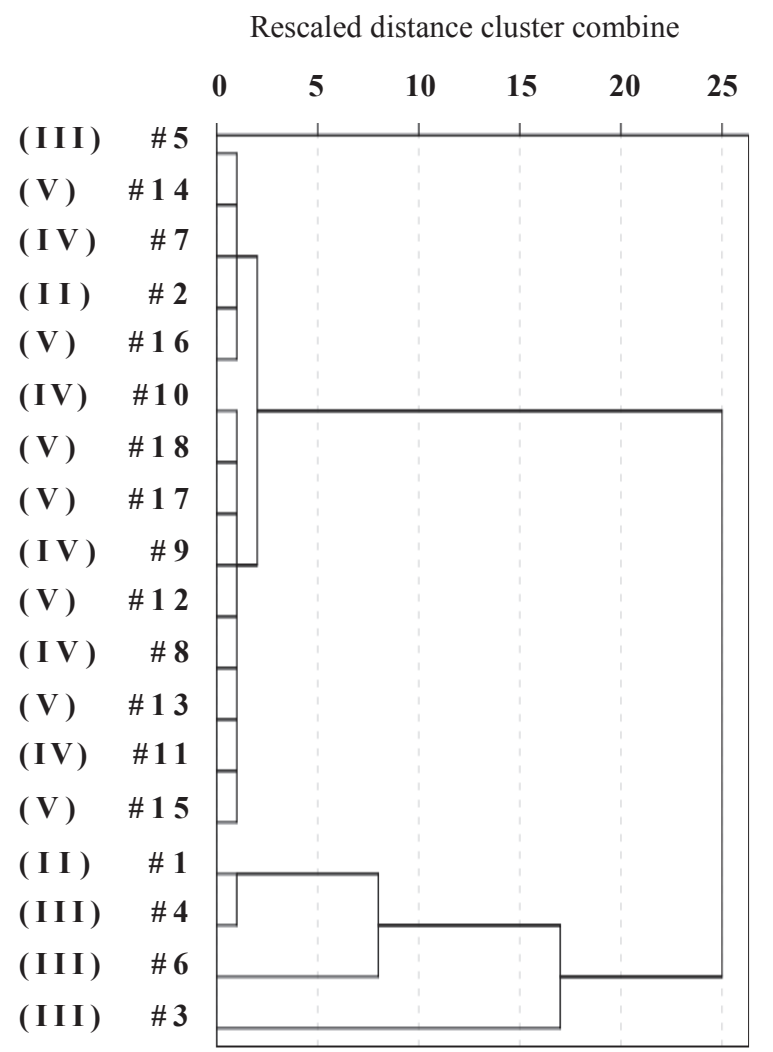

Fig. 3. Dendrogram generated from cluster analysis based on the PC1 and PC2 in Archicitrus.

Roman numbers in parentheses indicate Tanaka's category number. See Table 1 for the identification of numbers. of Sawamura et al. (1991). Nogata et al. (2006) separated 45 kinds of citrus fruits by PCA of flavonoids. Our results showed some similarity to their data, and it appears that the volatile components were associated in some way with flavonoids.

Cluster analysis The dendrogram generated by cluster analysis based on PC1 and PC2 in Archicitrus is shown in Fig. 3. Citron (\#3), Lumie (\#6), Tahiti lime (\#1) and Eureka lemon (\#4) are separated from other species. Species of Cephalocitrus, except Hirado buntan (\#7), and those of Aurantium, except Valencia (\#14) and Iyo (\#16), are close to each other

The dendrogram generated by cluster analysis based on PC1 and PC2 in Metacitrus and Fortunella is shown in Fig. 4. The species in these sections are roughly classified into three groups (C-1, C-2 and C-3). The C-1 includes Yuzu (\#19) of Osmocitrus and the Acumen Microacumen Citroidora Microcarpa samples, except for Tachibana (\#30) and Shiikuwasha (\#33). C-2 includes Kabosu (\#20) of Osmocitrus, the Fortunella, the Acumen Euacrumen samples and the Acrumen Microacrumen Citroidora Megacarpa samples. C-3 includes Yatsushiro (\#23), Oto (\#25), Keraji (\#24), Shiikuwasha (\#33) and Tachibana (\#30), and is separated from C-1 and C-2

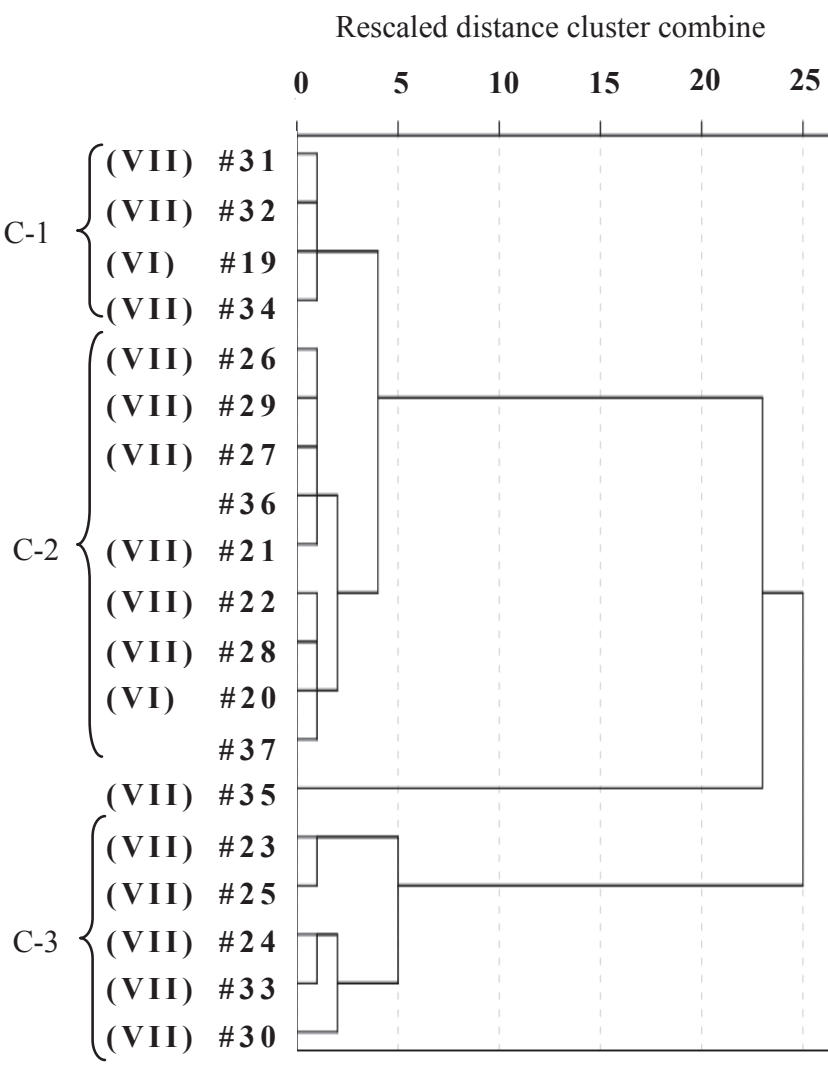

Fig. 4. Dendrogram generated from cluster analysis based on the PC1 and PC2 in Metacitrus and Fortunella.

Roman numbers in parentheses indicate Tanaka's category number. See Table 1 for the identification of numbers. 
groups. Shikikitsu (\#35) is distinguished from other species.

In conclusion, the composition of volatile components in citrus species revealed their characteristics and the correlation among samples. The composition of volatile components in citrus juices classified by Tanaka's system was consistent with each section, in particular, in the case of dividing the Citrus genus into the two subgenera Archicitrus and Metacitrus.

\section{References}

Attaway, J.A., Pieringer, A.P. and Barabas, L.J. (1967). The origin of citrus flavor components III. A study of the percentage variations in peel and leaf oil terpenes during one season. Phytochemistry, 6, 25-32.

Akakabe, Y., Sakamoto, M., Ikeda, Y. and Tanaka, M. (2008). Identification and characterization of volatile components of Japanese sour citrus fruits Citrus nagato-yuzukichi Tanaka. Biosci. Biotechnol. Biochem., 72, 1965-1968.

Akiyama, M., Murakami, K., Ikeda, M., Iwatsuki, K., Kokubo, S., Wada, A., Tokuno, K., Onishi, M., Iwabuchi, H. and Tanaka, K. (2005). Characterization of flavor compounds released during grinding of roasted robusta coffee beans. Food Sci. Technol. Res., 11, 298-307.

Berlinet, C., Guichard, E., Fournier, N. and Ducruet, V. (2007). Effect of pulp reduction and pasteurization on the release of aroma compounds in industrial orange juice. J. Food Sci., 72, 535-543.

Braddock, R.J., Goodrich, R.M. and Bryan, C.R. (2004). Variables affecting solid-phase microextraction headspace analysis of orange juice volatiles. Food Sci. Technol., 131, 423-436.

Choi, H.-S. (2005). Characteristic odor components of kumquat (Fortunella japonica Swingle) peel oil. J. Agric. Food. Chem., 53, 1642-1647.

Dharmawan, J., Kasapis, S., Curran, P. and Johnson, J.R. (2007). Characterization of volatile compounds in selected citrus fruits from Asia. Part 1: freshly-squeezed juice. Flavour Fragr. J., 22, 228-232.

González-Mas, M.C., Rambla, J.L., Alamar, M.C., Gutierres, A. and Granell, A. (2011). Comparative analysis of the volatile fraction of fruit juice from different Citrus species. PLoS One, 7, e22016.

Hashimoto, A., Kozima, K. and Sakamoto, K. (2006). Validity of SPME cryofocusing in analysis of volatile components in orange juice. Food Sci. Technol. Res., 12, 295-298.

Inafuku-Teramoto, S., Suwa, R., Furukawa, Y. and Kawamitsu, Y. (2011). Polymethoxyflavones, synephrine and volatile constitution of peels of citrus fruit grown in Okinawa. J. Japan. Soc.
Hort. Sci., 80, 214-224.

Jia, M., Zhang, Q.H. and Min, D.B. (1998). Optimization of solidphase microextraction analysis for headspace flavor compounds of orange juice. J. Agric. Food Chem., 46, 2744-2747.

Jordán, M., Goodner, K.L., Castillo, M. and Laencina, J. (2005). Comparison of two headspace solid phase microextraction fibers for the detection of volatile chemical concentration changes due to industrial processing of orange juice. J. Sci. Food Agric., 85, 1065-1071.

Kawaii, S., Tomono, Y., Katase, E., Ogawa, K. and Yano, M. (1999). Quantitation of flavonoid constituents in Citrus fruits. J. Agric. Food Chem., 47, 3565-3571.

Kawaii, S., Tomono, Y., Katase, E., Ogawa, K., Yano, M., Koizumi, M., Ito, C. and Furukawa, H. (2000). Quantitative study of flavonoids in leaves of Citrus plants. J. Agric. Food Chem., 48, 38653871.

Lota, M.-L., de Rocca Serra, D., Tomi, F., Jacquemond, C. and Casanova, J. (2002). Volatile components of peel and leaf oils of lemon and lime species. J. Agric. Food. Chem., 50, 796-805.

Mahattanatawee, K., Rouseff, R., Valim, M. F. and Naim, M. (2005). Identification and aroma impact of norisoprenoids in orange juice. J. Agric. Food Chem., 53, 393-397.

Nogata, Y., Sakamoto, K., Shiratsuchi, H., Ishi, T., Yano, M. and Ohta, H. (2006). Flavonoid composition of fruit tissues of citrus species. Biosci. Biotechnol. Biochem., 70, 178-192.

Sawamura, M., Kuwahara, S., Shichiri, K. and Aoki, T. (1990). Volatile constituents of several varieties of pummelos and a comparison of the nootkatone levels in pummelos and other citrus fruits. Agric. Biol. Chem., 54, 803-805.

Sawamura, M., Shichiri, K., Ootani, Y. and Zheng, X.H. (1991). Volatile constituents of several varieties of pummelos and characteristics among citrus species. Agric. Biol. Chem., 55, 2571-2578.

Steffen, A. and Pawliszyn, J. (1996). Analysis of flavor volatiles using headspace solid-phase microextraction. J. Agric. Food Chem., 44, 2187-2193.

Tamura, H., Padrayuttawat, A. and Tokunaga, T. (1999). Seasonal change of volatile compounds of Citrus sudachi during maturation. Food Sci. Technol. Res., 5, 156-160.

Tanaka, T. (1969). Misunderstanding with regards citrus classification and nomenclature. Bull. Univ. Osaka Pref., Ser. B., 21, 139145.

Wu, C.-M. and Wang, Z. (2000). Volatile compounds in fresh and processed Shiitake mushrooms (Lentinus edodes Sing.). Food Sci. Technol. Res., 6, 166-170. 